\title{
MicroRNA-197 reverses the drug resistance of fluorouracil-induced SGC7901 cells by targeting mitogen-activated protein kinase 1
}

\author{
HAI-LIN XIONG, SI-WEI ZHOU, AI-HUA SUN，YING HE，JUN LI and XIA YUAN \\ Department of Medical Oncology, Huizhou Municipal Central Hospital of Guangdong Province, \\ Huizhou, Guangdong 516000, P.R. China
}

Received March 5, 2014; Accepted April 10, 2015

DOI: $10.3892 / \mathrm{mmr} .2015 .4052$

\begin{abstract}
MicroRNAs (miRNAs) are a group of small non-coding RNA molecules, which serve an important function in the development of multidrug resistance in cancer through the post-transcriptional regulation of gene expression and RNA silencing. In the present study, the functional effects of miR-197 were analyzed in chemo-resistant gastric cancer cells. Low expression levels of miR-197 were observed in the fluorouracil (5-FU)-resistant gastric cell line SGC7901/5-FU when compared with those in the parental gastric cell line SGC7901. Overexpression of miR-197 in SGC7901/5-FU cells was identified to partially restore 5-FU sensitivity. miRNA target prediction algorithms suggested that mitogen-activated protein kinase 1 (MAPK1) is a candidate target gene for miR-197. A luciferase reporter assay confirmed that miR-197 led to silencing of the MAPK1 gene by recognizing and then specifically binding to the predicted site of the MAPK1 mRNA 3'-untranslated region. When miR-197 was overexpressed in SGC7901 cells, the protein levels of MAPK1 were downregulated. Furthermore, MAPK1 knockdown significantly increased the growth inhibition rate of the SGC7901/5-FU cells compared with those in the control group. These results indicated that miR-197 may influence the sensitivity of 5-FU treatment in a gastric cancer cell line by targeting MAPK1.
\end{abstract}

\section{Introduction}

Gastric cancer is prevalent worldwide and although the incidence and mortality have markedly fallen, it remains the fourth most common cancer and the second leading cause of cancer-associated mortality (1). In spite of early use of

Correspondence to: Dr Xia Yuan, Department of Medical Oncology, Huizhou Municipal Central Hospital of Guangdong Province, 41 North Eling Road, Huizhou, Guangdong 516000, P.R. China E-mail: yuanxia20140101@163.com

Key words: gastric cancer, microRNA-197, 5-fluorouracil resistance, mitogen-activated protein kinase 1 adjuvant chemotherapy after surgery, poor outcomes following surgical resection and high recurrence rates remain, making gastric cancer challenging to treat. Compared with no adjuvant treatment, combination-chemotherapy has been reported to significantly prolong the disease-free period and the patients' survival rate five years subsequent to surgery $(2,3)$. The occurrence of resistance is commonly observed during the course of chemotherapy treatment. The efficacy of chemotherapy is limited by simultaneous resistance to multiple drugs that are neither mechanistically nor structurally linked. In the resistance of cancer cells to chemotherapeutic agents, DNA methylation, gene mutation and histone modification serve important roles (2,3). In 2007, Climent et al (4) observed that the deletion of chromosome 11q, which carries the region containing the microRNA (miR)-125b gene, may contribute to the sensitivity of patients with breast cancer to anthracycline-based chemotherapy. This suggested a possible link between miRNA dysregulation and chemotherapy resistance.

miRNAs regulate gene expression in multicellular organisms by post-transcriptionally affecting the stability and translation of mRNAs (5), which are transcribed by RNA polymerase II or III in the nucleus (6). The primary capped and polyadenylated transcripts (pri-miRNA) are cleaved by the Drosha ribonuclease III enzyme to produce a $\sim 70$-nucleotide stem-loop precursor miRNA (pre-miRNA) (7-9). Pre-miRNA is transported to the cytoplasm by exportin 5 and is then processed into mature miRNAs by the RNase III enzyme Dicer $(10,11)$. Mature miRNA is incorporated into an RNA-induced silencing complex (RISC), which imperfectly pairs with the 3'-untranslated region (3'UTR) of the target gene mRNA. As a result, the translation of the target gene mRNAs is inhibited or destabilized (12-14). Previous studies indicated critical functions of miRNAs in diverse biological processes, including tumor angiogenesis, proliferation, cell differentiation, apoptosis, adhesion and metastasis of tumor cells (15-19) and cancer chemotherapy multidrug resistance (MDR) (20). Therefore, elucidation of the regulatory role of miRNAs may provide a novel understanding of the molecular events in various biological processes, and suggest that abnormally expressed miRNAs in various types of human cancer serve as oncogenes or tumor suppressor genes by targeting transcripts of essential protein coding genes in tumorigenesis. 
Previous studies $(21,22)$ have suggested that, in addition to oncogenesis, the different expression levels of certain miRNAs are associated with the response to chemotherapeutic agents. Chemotherapy is frequently unsuccessful due to either intrinsic or acquired MDR of cancer cells following an initial round of treatment (23). Zhu et al (24) demonstrated that the MDR cancer cell lines A2780DX5 and KB-V1 exhibited higher expression levels of miR-27a and miR-451 than their parental lines A2780 and KB-3-1. Downregulation of miR-27a or miR-451 expression has been reported to reduce the expression levels of P-glycoprotein (P-gp) and MDR1 mRNA. The intracellular accumulation of cytotoxic drugs due to being transported by P-gp was enhanced by the treatment with the anti-miR-27a or anti-miR-451 (24). Xia et al (22) analyzed the possible role of miRNAs in the development of MDR in gastric cancer cells. They identified that miR-15b and miR-16 were downregulated in the MDR gastric cancer cell line SGC7901/VCR compared with that in the control group. In addition, overexpression of miR-15b or miR-16 has been reported to sensitize SGC7901/VCR cells to vincristine, doxorubicin, etoposide and cisplatin in an in vitro drug sensitivity assay. By contrast, inhibition of miR-15b or miR-16 expression may contribute to MDR in SGC7901 cells. Meng et al (25) also indicated that miR-21, miR-141 and miR-200b were dysregulated in malignant cholangiocytes. Downregulation of miR-21 and miR-200b increased sensitivity to gemcitabine, whereas inhibition of miR-141 reduced cell growth.

As described above, miRNAs serve as regulators of gene expression and may influence the response of cancer cells to chemotherapy. Thus, in the present study, the expression levels of miR-197 were investigated in the fluorouracil (5-FU)-resistant human gastric cancer cell line SGC7901/5-FU and its parental cell line SGC7901. The present study focused on the effects of miR-197 on 5-FU drug resistance in SGC7901 gastric cancer cells in addition to the identification of its direct target gene. It was hypothesized that miR-197 may present a novel therapeutic for preventing resistance against 5-FU by targeting the expression of resistance-associated genes in patients with gastric carcinoma.

\section{Materials and methods}

Cell culture and transfection. Cells of the human gastric cancercell line SGC-7901 (American Type Culture Collection, Manassas, VA, USA) were cultured in RPMI-1640 medium (Gibco-BRL, Invitrogen Life Technologies, Carlsbad, CA, USA), which was supplemented with $10 \%$ heat-inactivated fetal bovine serum, $100 \mathrm{IU}$ penicillin $/ \mathrm{ml}$ and $100 \mu \mathrm{g} / \mathrm{ml}$ streptomycin (Invitrogen Life Technologies). All of the cells were maintained in a humidified $5 \%(\mathrm{v} / \mathrm{v})$ atmosphere of $\mathrm{CO}_{2}$ at $37^{\circ} \mathrm{C}$. The 5 -FU-resistant variant SGC7901/5-FU was obtained from the parent cell line (SGC-7901) by step by step exposure to increasing concentrations of 5-FU $(5,10$, 20 and $40 \mathrm{mg} / \mathrm{ml}$ in three-day intervals; Sigma-Aldrich, St. Louis, MO, USA). To maintain the 5-FU-resistant phenotype, 5-FU was added to the culture media with a final drug concentration of $1 \mathrm{mg} / \mathrm{ml} 5$-FU for SGC7901/5-FU cells. Lipofectamine 2000 reagent (Invitrogen Life Technologies) was used for the cell transfection in accordance with the manufacturer's instructions.
Luciferase reporter assay. The gastric cancer cells were co-transfected with miR-197 and MAPK1 3'UTR or MAPK1 3'UTR-mutant (Zhiyou Bio Company, Guangzhou, China) in 48-well plates. The vector pDsRed2-N1 (Clontech Laboratories, Inc., Mountain View, CA, USA) expressing red fluorescent protein (RFP) was transfected and used for normalization. The intensities of enhanced green fluorescent protein (EGFP) and RFP fluorescence were measured using a F-4500 Fluorescence Spectrophotometer (Hitachi, Ltd., Tokyo, Japan).

Reverse transcription-quantitative polymerase chain reaction $(R T-q P C R)$. RT-qPCR was performed in order to detect the relative levels of the transcripts. The large RNA and small RNA of tissue samples were isolated using an mirVana ${ }^{\mathrm{TM}}$ miRNA Isolation kit (Ambion Life Technologies, Carlsbad, CA, USA) according to the manufacturer's instructions. The transcripts were generated from $2 \mu \mathrm{g}$ RNA extracted from the cells through reverse transcription using Moloney murine leukemia virus reverse transcriptase (Promega Corporation, Madison, WI, USA). The $\beta$-actin gene was used as an internal control standard for the PCR reaction. PCR was performed under the following conditions: $94^{\circ} \mathrm{C}$ for $4 \mathrm{~min}$, followed by 40 cycles of $94^{\circ} \mathrm{C}$ for $1 \mathrm{~min}, 56^{\circ} \mathrm{C}$ for $1 \mathrm{~min}$ and $72^{\circ} \mathrm{C}$ for $1 \mathrm{~min}$. PCR was performed in a total volume of $50 \mu \mathrm{l}$ with AmpliTaq Gold 360 DNA Polymerase (Applied Biosystems, Foster City, CA, USA). After CE on an ABI 3130 Genetic Analyzer (Applied Biosystems), data were collected using GeneMapper v4.0 software for fragment analysis (Applied Biosystems).

MTT assay. SGC-7901 and SGC-7901/5-FU cells were respectively seeded into a 96 -well plate at a denstiy of 8,000 cells/well one day prior to transfection. The cells were transfected with anti-miR-197, miR-197 or the control vector $(0.15 \mu \mathrm{g} / \mathrm{well}$; Shanghai GenePharma Co., Ltd., Shanghai, China). 5-FU was then added so that the final concentrations were $0.5,1,2$, 4 and $8 \mu \mathrm{mol} / \mathrm{l}$. Following $48 \mathrm{~h}$ of incubation, the MTT assay was used to evaluate the cell viability. MTT solution $(20 \mu \mathrm{l}$; Sigma-Aldrich) was added to $100 \mu \mathrm{l}$ culture media and cells were incubated for a further $4 \mathrm{~h}$ at $37^{\circ} \mathrm{C}$ The growth inhibition rate was calculated as: Growth inhibition $(\%)=(1-\mathrm{A}$ of experimental group/A of control group) x100, where A represents the absorbance at $570 \mathrm{~nm}$, which was measured using a $\mu$ Quant Universal Microplate Spectrophotometer (BioTek Instruments, Inc., Winooski, VT, USA).

Western blot analysis. Cultured cells were lysed using radioimmunoprecipitation assay buffer (0.1\% SDS, $1 \%$ Triton $\mathrm{X}-100,1 \mathrm{mM} \mathrm{MgCl}{ }_{2}$ and $10 \mathrm{mM}$ Tris-HCl; $\mathrm{pH} 7.4$; all from Sigma-Aldrich) in $4^{\circ} \mathrm{C}$ for $30 \mathrm{~min}$. The protein extracts $(50 \mu \mathrm{g})$ were fractionated by $10 \%$ SDS-PAGE and then transferred onto nitrocellulose membranes. The following antibodies were used: Anti-MAPK1 (sc-136288; species, mouse; 1:500) and anti-GAPDH (sc-365062; species, mouse; 1:1,000) (Santa Cruz Biotechnology, Inc., Dallas, TX, USA). The bound antibodies were detected using the ECL Plus Western Blotting Detection system (GE Healthcare, Little Chalfont, UK) and the chemiluminescent signals were detected with the using high-performance chemiluminescence film (GE Healthcare). 
A

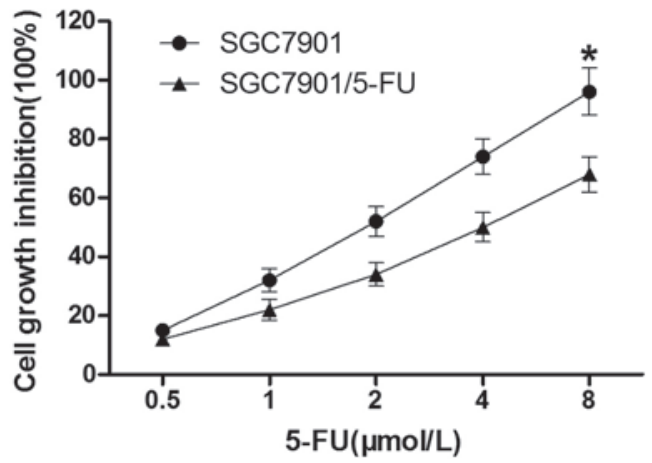

B

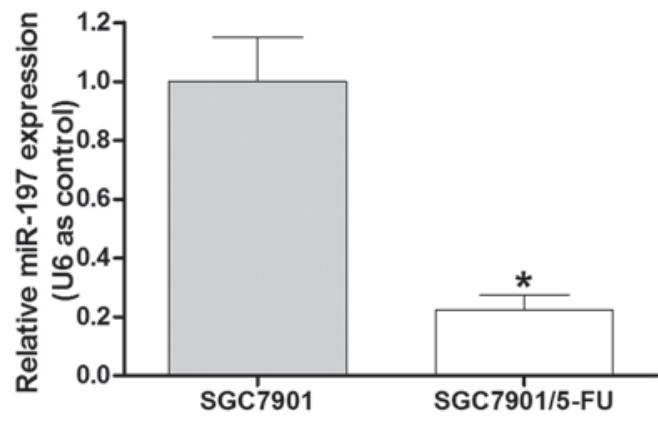

Figure 1. (A) Cell growth inhibition rate of SGC7901 and SGC7901/5-FU cells. (B) Expression levels of miR-197 in SGC7901 and SGC7901/5-FU cells. The cells were treated with various doses of 5-FU $(0.5,1.0,2.0,4.0$ and $8.0 \mu \mathrm{mol} / \mathrm{l})$. After $48 \mathrm{~h}$ of transfection, an MTT assay was performed to assess the growth inhibition rate of the cells. $\mathrm{P}<0.05$ vs. control. 5-FU, fluorouracil; miR-197, microRNA-197; SGC7901/5-FU, SGC7901 cell line resistant to 5-FU.

Small interfering (si)RNA transfection. According to the manufacturer's instructions, the SignalSilence MAPK1 siRNA kit (Cell Signaling Technology, Inc., Danvers, MA, USA) was used to silence MAPK1 protein expression. Subsequent to transfection for $48 \mathrm{~h}$, siRNA transfection efficiency was measured using western blot analysis.

Flow cytometric analysis. In order to synchronize the cell growth, the transfected cells were maintained in serum-free culture medium for starvation. After $24 \mathrm{~h}$, one group of cells was harvested and the other was cultured in complete medium for $24 \mathrm{~h}$. The harvested cells were fixed in $95 \%$ ethanol and stored at $-60^{\circ} \mathrm{C}$. The fixed cells were washed with phosphate-buffered saline, re-suspended in propidium iodine (PI) staining buffer (Bio-Light, Shanghai, China), which contained $50 \mathrm{mg} / \mathrm{ml} \mathrm{PI}, 50 \mathrm{mg} / \mathrm{ml}$ Annexin V, and 50mg/ml RNaseA, and were then incubated at $4^{\circ} \mathrm{C}$ for $30 \mathrm{~min}$ in the dark. The samples were analyzed using a FACScan flow cytometer and Cell Quest 2.0 analysis software (BD Biosciences, San Jose, CA, USA).

Statistical analysis. Values are expressed as the mean \pm standard deviation. A two-tailed Student's t-test was used for comparison and $\mathrm{P}<0.05$ was considered to indicate a statistically significant difference. GraphPad 5.0 (GraphPad, Inc., La Jolla, CA, USA) was used for all statistical analyses.

\section{Results}

miR-197 is downregulated in 5-FU-resistant SGC7901/5-FU cells. To detect the cell growth inhibition rate of the SGC7901/5-FU cell line and the parental SGC7901 cell line, an MTT assay was performed (Fig.1A). The results demonstrated that compared with the SGC7901 cell line, the SGC7901/5-FU cells exhibited clear resistance to 5-FU. In order to determine the role of miR-197 in the 5-FU-resistant gastric cancer cell line SGC7901/5-FU, RT-qPCR analysis was conducted. The results indicated that miR-197 was downregulated in SGC7901/5-FU cells (Fig.1B), which suggested that miR-197 may contribute to 5-FU drug resistance in gastric cancer cells.

Knockdown of miR-197 leads to resistance to 5-FU in the SGC7901 cells. To investigate the association between
miR-197 and 5-FU resistance in SGC7901 cells, the effect of downregulation of miR-197 in 5-FU-sensitive SGC7901 cells was assessed. A commercially synthesized miR-197 inhibitor (anti-miR-197) was used to alter the miR-197 expression levels in SGC7901 cells. The validity of miR-197 ectopic expression was confirmed by RT-qPCR following transfection with anti-miR-197 or the control vector into SGC7901 cells. The results indicated that the commercially synthesized anti-miR-197 was able to reduce miR-197 expression in SGC7901 cells compared with that in the control group $(\mathrm{P}<0.05$; Fig. 2A). The group transfected with anti-miR-197 was observed to have a significantly higher survival rate than the control group (Fig. 2B). These results suggested that downregulation of miR-197 led to 5-FU resistance of SGC7901 cells.

Overexpression of miR-197 may partially enhance 5-FU sensitivity in SGC7901/5-FU cells. To further determine the effects of miR-197 overexpression in SGC7901/5-FU cells, miR-197 mimics or the control vector were transfected into SGC7901/5-FU cells. RT-qPCR analysis confirmed that the miR-197 mimics were able to increase miR-197 expression in SGC7901/5-FU cells compared with that in the control group ( $\mathrm{P}<0.05$; Fig. 2C). The SGC7901/5-FU cells which were transfected with the miR-197 mimics exhibited a significantly lower survival rate than the cells in the negative control group following 5-FU exposure (Fig. 2D). These results suggested that the overexpression of miR-197 may sensitize the SGC7901/5-FU cells to 5-FU.

miR-197 directly targets the MAPK1 3'UTR in SGC7901 gastric cancer cells. To determine the candidate target gene of miR-197, a bioinformatics analysis using Targetscan 6.2 (http:// www.targetscan.org/) was conducted to predict potential target genes. It was observed that the miR-197 complementary binding sites were contained in the 3'UTR of MAPK1 mRNA (Fig. 3A). A luciferase reporter assay was performed to validate that MAPK1 can be directly targeted by miR-197 using engineered EGFP reporter vectors that had either the wild-type 3'UTR of MAPK1 or the mutant UTR with a 5-base mutation in the complementary seed sequence (Fig. 3A). pDsRed2-NI was also co-transfected for normalization. SGC7901 cells were co-transfected with MAPK1 3'UTR and miR-197 or 
A

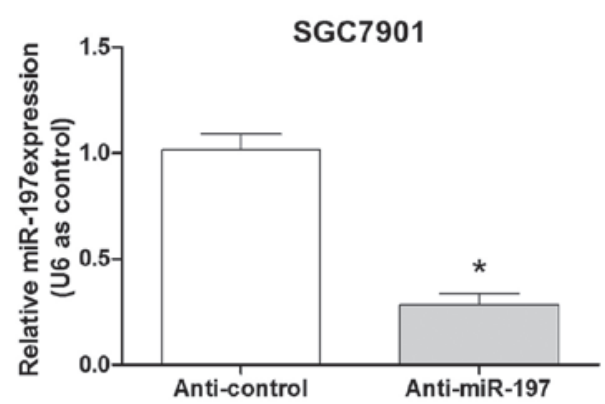

C

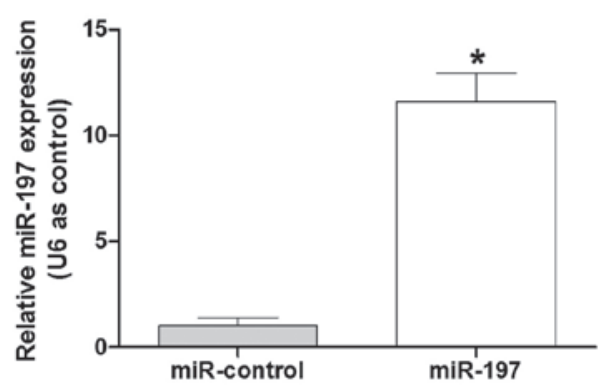

B

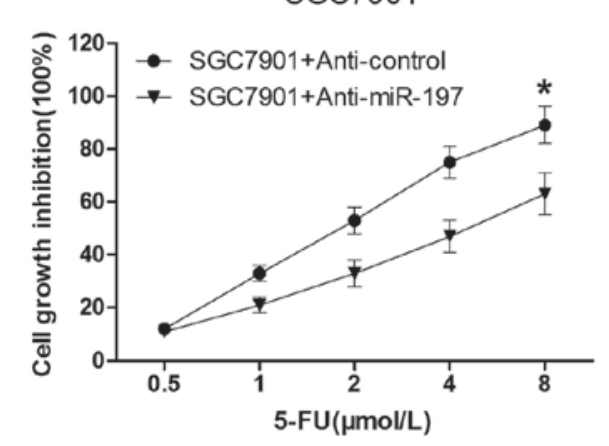

D

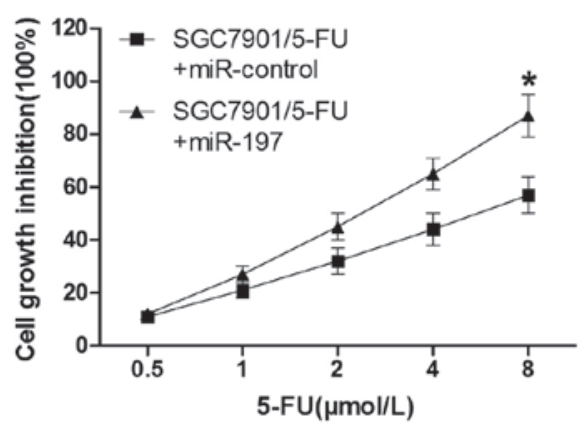

Figure 2. Expression levels of miR-197 affect the sensitivity of SGC7901 cells to 5-FU. (A) Following transfection with anti-miR-197, the expression levels of miR-197 were significantly reduced in SGC7901 cells. (B) Following transfection with anti-miR-197 or control, SGC7901 cells were treated with various doses of 5-FU (0.5, 1.0, 2.0, 4.0 or $8.0 \mu \mathrm{mol} / \mathrm{l})$ for $48 \mathrm{~h}$. An MTT assay was performed to determine the cell growth inhibition. (C) Following transfection of the miR-197 mimics, the expression levels of miR-197 were significantly increased in SGC7901/5-FU cells. (D) Following transfection with the miR-197 mimics or control, the SGC7901/5-FU cells were treated with various doses of 5-FU $(0.5,1.0,2.0,4.0$ and $8.0 \mu \mathrm{mol} / 1)$ for $48 \mathrm{~h}$. The MTT assay was performed to determine the cell growth inhibition. Values are expressed as the mean \pm standard deviation $(n=3)$. "P $<0.05$ vs. control. miR-197, microRNA-197; 5-FU, fluorouracil; SGC7901/5-FU, SGC7901 cell line resistant to 5-FU.

A

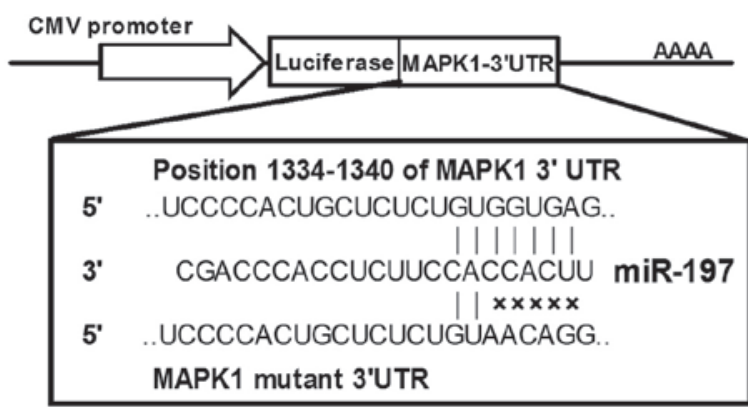

C

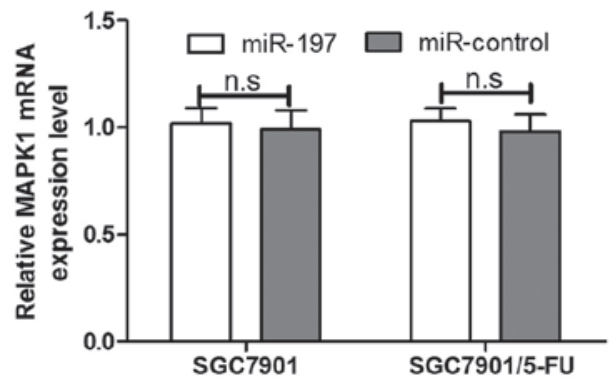

B
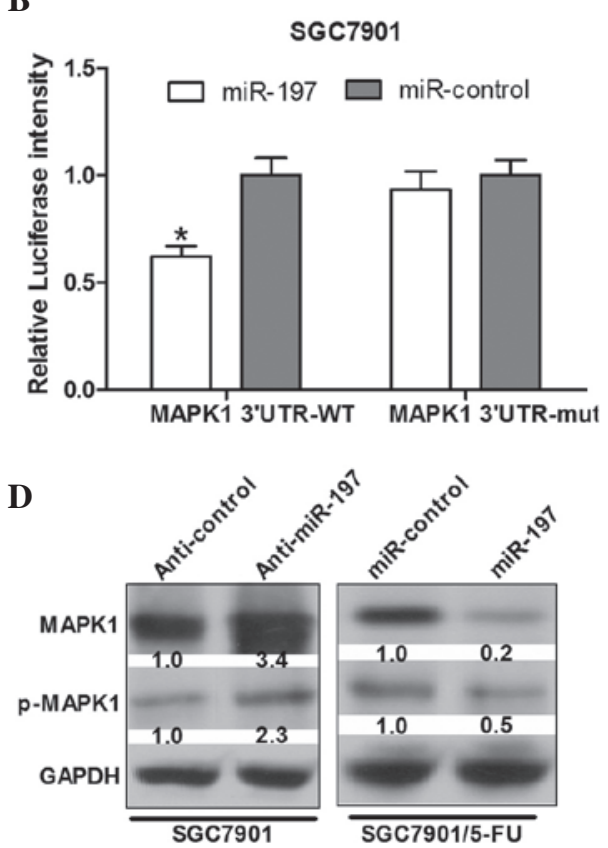

Figure 3. MAPK1 is a direct target of miR-197. (A) Predicted binding sites of miR-197 on MAPK1 mRNA. The mutant UTR contains 5 bases in the complementary seed sequences. (B) miR-197 mimics transfected together with luciferase reporter vector pGL3-MAPK1 3'UTR or the mutant vector pGL3-MAPK1 3'UTR mut into SGC7901 cells. The relative luciferase intensity was significantly different between the miR-197 and miR-control group. Values are expressed as the mean \pm standard deviation $(n=3)$. ${ }^{*} \mathrm{P}<0.05$. (C) Reverse transcription-quantitative polymerase chain reaction was conducted to detect the expression levels of MAPK1 mRNA in SGC7901 and SGC7901/5-FU cells which were transfected with the miR-197 mimics or control. Values are expressed as the mean \pm standard deviation (n=3). (D) MAPK1 and p-MAPK1 protein levels in the SGC7901 cells transfected with the miR-197 inhibitor or control, and SGC7901/5-FU cells transfected with the miR-197 mimics or control. p-MAPK1, phosphorylated mitogen-activated protein kinase 1; miR-197, microRNA-197; UTR, untranslated region; 5-FU, fluorouracil; WT, wild-type; mut, mutated; SGC7901/5-FU, SGC7901 cells resistant to 5-FU. 
A

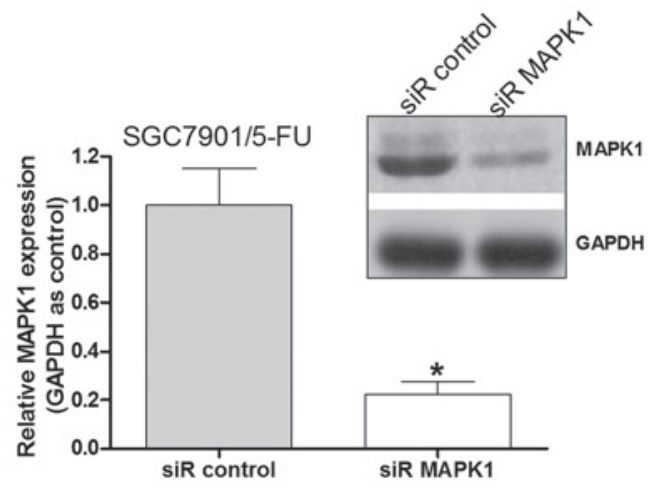

B

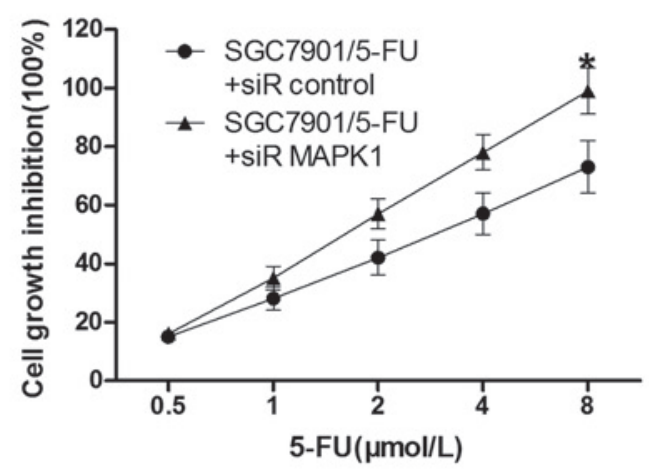

C SGC7901/5-FU+control

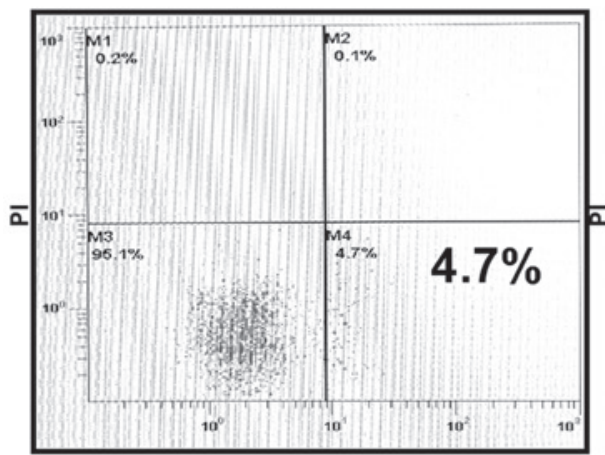

Annexin V

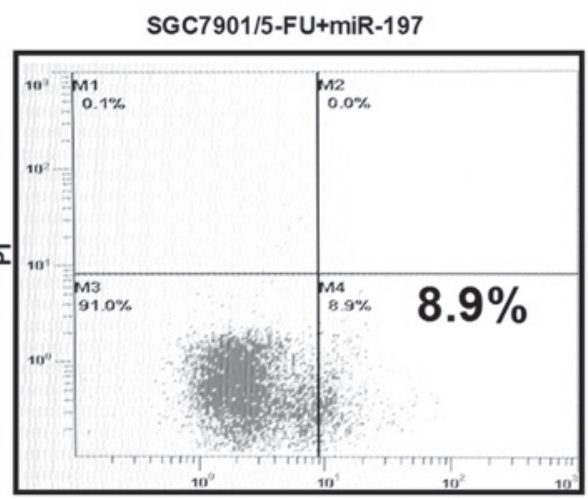

Annexin V
SGC7901/5-FU+siR MAPK1

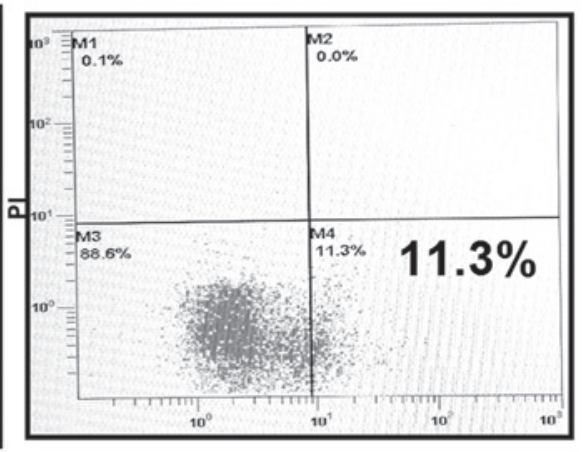

Annexin V

Figure 4. MAPK1 is important in 5-FU resistance in SGC7901/5-FU cells. (A) MAPK1 protein levels in the SGC7901/5-FU cells following treatment with MAPK1 siRNA or a scrambled siRNA. (B) SGC7901/5-FU cells were treated with various doses of 5-FU (0.5, 1.0 and $2.0 \mu$ mol/1) following $48 \mathrm{~h}$ of transfection. The cell growth inhibition rate was determined using an MTT assay. (C) Apoptosis detected by flow cytometry. SGC-7901/5-FU cells were untreated, transfected with miR-197 mimics or transfected with siRNA MAPK1 for $48 \mathrm{~h}$, and apoptotic cells were detected by flow cytometry. Values are expressed as the mean \pm standard deviation $(n=3)$. *P<0.05 vs. control. MAPK1, mitogen-activated protein kinase 1; 5-FU, fluorouracil; SGC7901/5-FU, SGC7901 cells resistant to 5-FU; siR/siRNA, small interfering RNA; PI, propidium iodide.

the control vector. Compared with the control vector, overexpression of miR-197 was able to significantly inhibit EGFP expression ( $\mathrm{P}<0.05)$ (Fig. 3B). By contrast, EGFP expression levels of cells transfected with a mutant of the MAPK1 3'UTR binding site were not influenced by overexpression of miR-197 (Fig. 3B), indicating that miR-197 was able to complementarily bind to the specific sites of the MAPK1 mRNA 3'UTR and negatively regulate the expression of the MAPK1 gene.

miR-197 negatively regulates MAPK1 at the post-transcriptional level. Downregulation of gene expression is the main function of miRNAs, which occurs through translational repression, cleavage of mRNA or in a variety of other processes by binding to 3'UTRs of target genes. To examine whether miR-197 suppresses endogenous MAPK1 expression, SGC7901 and SGC791/5-FU cells were transfected with anti-miR-197 and miR-197 mimics, and MAPK1 protein expression was assessed via western blot analysis.

The results demonstrated that overexpression of miR-197 reduced the expression levels of MAPK1 and the phosphorylation of MAPK1 protein by 80 and 50\%, respectively, in SGC7901/5-FU cells (Fig. 3D). However, upregulation of miR-197 in SGC7901/5-FU cells was not able to reduce endogenous MAPK1 mRNA according to RT-qPCR analysis (Fig. 3C). In addition, following transfection with
anti-miR-197, significant increases were observed in the endogenous MAPK1 protein expression levels and the phosphorylation of MAPK1 in SGC7901 cells (Fig. 3D); however, MAPK1 mRNA levels remained unchanged (Fig. 3C). These results suggested an inverse correlation between miR-197 and MAPK1 protein expression levels.

MAPK1 is a crucial signaling factor in 5-FU resistance in SGC7901/5-FU cells. To validate whether MAPK1 serves a role in miR-197-induced 5-FU resistance, the MAPK1 gene was silenced to a certain degree in the SGC7901/5-FU cells. Western blot analysis demonstrated that MAPK1 siRNA effectively reduced the MAPK1 protein levels (Fig. 4A). Following MAPK1 siRNA transfection, the cell growth inhibition rate by was then detected following exposure to various concentrations of 5-FU (0.5-8 $\mu \mathrm{M})$. Knockdown of MAPK1 significantly increased the growth inhibition rate of the SGC7901/5-FU cells compared with that in the control group (Fig. 4B). The SGC7901/5-FU cells transfected with miR-197 mimics in addition to those transfected with siR-MAPK1 demonstrated a significantly increased apoptotic rate compared with that in the negative control group $(\mathrm{P}<0.05)$. These results suggested that miR-197 may improve the 5-FU sensitivity of the SGC7901/5-FU cells by downregulating MAPK1. 


\section{Discussion}

MDR is a multifactorial process, which is responsible for the lack of chemosensitivity in primary and secondary tumors (20). 5 -FU is the most commonly used chemotherapeutic drug in patients with gastric cancer, and one of the main causes of chemotherapeutic treatment failure in advanced gastric cancer is 5-FU resistance $(2,26)$. Therefore, the development of novel strategies enhancing the efficacy of 5-FU is essential for effective therapy. It is widely accepted that abnormal genetic expression and the alteration of the dynamic balance between oncogenes and tumor suppressor genes commonly leads to the formation of tumors (27). miRNAs, in addition to other genes, participate in the regulation of oncogenes and tumor suppressor genes (5). Increasing numbers of studies have identified that abnormal miRNA expression is also responsible for the absence of a chemotherapeutic response. However, the role of miRNAs in drug-resistance of gastric cancer remains to be fully elucidated.

The present study aimed to investigate a novel miRNA, which was able to regulate MAPK1 protein expression levels through binding to specific sites of the MAPK1 3'UTR, and to validate its effects on 5-FU resistance using SGC7901 cells. The results showed that the suppression of MAPK1 in addition to miR-197 upregulation acted together to achieve sensitivity to the chemotherapeutic 5-FU. It was therefore suggested that altering miR-197 expression may improve the sensitivity of SGC7901 cells to 5-FU treatment.

Using RT-qPCR, it was identified that miR-197 exhibited lower expression levels in human 5-FU-resistant SGC7901/5-FU gastric cancer cells than that in the parental cell line SGC7901. The results suggested that miR-197 may be critical in the development of gastric cancer chemotherapy resistance. Therefore, it was hypothesized that miR-197 is an inhibitory factor of 5-FU resistance in gastric cancer cells due to the low expression levels of miR-197 in SGC7901/5-FU. The cell growth inhibition rate was assessed using the MTT assay in order to investigate the association between miR-197 and the growth inhibition capacity of gastric cancer cell lines with or without 5-FU resistance. Following transfection with anti-miR-197, the SGC-7901 cell growth inhibition rate was significantly reduced. In addition, compared with the control group, upregulation of miR-197 enhanced cell growth inhibition following 5-FU treatment. miR-197 is therefore closely associated with 5-FU resistance. Bioinformatics analysis was conducted to predict MAPK1 as one of the candidate target genes of miR-197, which was then confirmed by experimental evidence in the present study. Through complementarily binding to the 3'UTR of MAPK1 seed region, miR-197 was able to regulate MAPK1 protein expression directly. Overexpression of miR-197 resulted in a reduction in the fluorescence intensity of MAPK1-3'UTR reporter vector-transfected cells in the luciferase reporter assay (Fig. 3B). However, the 5-base mutation of the miR-197 binding sites in MAPK1-3'UTR abolished the effect of miR-197 on the regulation of EGFP fluorescence intensity (Fig. 3B). In addition, endogenous MAPK1 expression levels and the phosphorylation of MAPK1 protein were reduced in SGC7901/5-FU cells with the overexpression of miR-197 and in SGC7901 cells transfected with anti-miR-197 (Fig. 3D). The alteration of miR-197 expression levels was observed to not effect the MAPK1 mRNA levels. These results suggested that miR-197 regulated MAPK1 protein expression.

The MAPK cascade is one of the intracellular signal transduction pathways specific to the amino acids serine, threonine and tyrosine. MAPKs are present exclusively in eukaryotic cells, which are involved in direct cellular responses to various stimuli, including mitogens, hormones, cytokines and osmotic stress (28). Extracellular signal-regulated kinases (ERKs), c-Jun-N-terminal protein kinases and $\mathrm{p} 38$ are all involved in the MAPK pathway (29). MAPKs participate in gene expression, cell proliferation, differentiation, cell mitosis and apoptosis (28). Due to the integral role in the regulation of cancer cell proliferation, invasion and survival, the MAPK pathway is considered to be a potential target for therapeutic intervention in cancer. MAPK1 is also known as ERK2; ERK was the first MAPK family member to be identified, and it also serves as the key molecule in the MAPK signaling pathway. ERKs are responsible for transporting extracellular stimuli from the cell surface to the nucleus (30). In 2007, Katayama et al (31) demonstrated that the ERK pathway was able to positively regulate P-gp expression in the MDA-MB-231/MDR cell line, and that inhibition of the MAPK pathway can suppress cell surface P-gp expression by promoting its degradation. Furthermore, various studies have demonstrated that modulation of ERK activation may reverse MDR in prostatic, gastric and hematopoietic cancer (32-35).

Previous studies have suggested that modulation of ERK activation may provide a novel method to reverse MDR in cancer cells (36). In the present study, the association between miR-197 and MAPK1 in gastric cancer cells was investigated, and it was confirmed that miR-197 reduced cell survival and 5-FU resistance in human gastric cancer by directly targeting MAPK1. It was also identified that knockdown of MAPK1 improved the 5-FU sensitivity of SGC7901/5-FU cells, which is consistent with the results of miR-197 overexpression. These results indicated that miR-197 mimics may be used as a therapeutic approach in 5-FU-resistant gastric cancer.

\section{References}

1. Murray CJ and Lopez AD: Alternative projections of mortality and disability by cause 1990-2020: Global Burden of Disease Study. Lancet 349: 1498-1504, 1997.

2. Cunningham D, Allum WH, Stenning SP, et al: MAGIC Trial Participants: Perioperative chemotherapy versus surgery alone for resectable gastroesophageal cancer. N Engl J Med 355: 11-20, 2006.

3. Rivera F, Vega-Villegas ME and López-Brea MF: Chemotherapy of advanced gastric cancer. Cancer Treat Rev 33: 315-324, 2007.

4. Climent J, Dimitrow P, Fridlyand J, Palacios J, Siebert R, Albertson DG, Gray JW, Pinkel D, Lluch A and Martinez-Climent JA: Deletion of chromosome 11q predicts response to anthracycline-based chemotherapy in early breast cancer. Cancer Res 67: 818-826, 2007.

5. Bartel DP: MicroRNAs: Genomics, biogenesis, mechanism, and function. Cell 116: 281-297, 2004.

6. Lee Y, Kim M, Han J, Yeom KH, Lee S, Baek SH and Kim VN: MicroRNA genes are transcribed by RNA polymerase II. EMBO J 23: 4051-4060, 2004.

7. Han J, Lee Y, Yeom KH, Kim YK, Jin H and Kim VN: The Drosha-DGCR8 complex in primary microRNA processing. Genes Dev 18: 3016-3027, 2004.

8. Lee Y, Ahn C, Han J, et al: The nuclear RNase III Drosha initiates microRNA processing. Nature 425: 415-419, 2003.

9. Lee Y, Jeon K, Lee JT, Kim S and Kim VN: MicroRNA maturation: Stepwise processing and subcellular localization. EMBO J 21: 4663-4670, 2002. 
10. Lund E, Güttinger S, Calado A, Dahlberg JE and Kutay U: Nuclear export of microRNA precursors. Science 303: 95-98, 2004.

11. Hutvágner G, McLachlan J, Pasquinelli AE, Bálint E, Tuschl T and Zamore PD: A cellular function for the RNA-interference enzyme Dicer in the maturation of the let-7 small temporal RNA. Science 293: 834-838, 2001.

12. Parker JS and Barford D: Argonaute: A scaffold for the function of short regulatory RNAs. Trends Biochem Sci 31: 622-630, 2006.

13. Peters L and Meister G: Argonaute proteins: Mediators of RNA silencing. Mol Cell 26: 611-623, 2007.

14. Pillai RS, Bhattacharyya SN and Filipowicz W: Repression of protein synthesis by miRNAs: How many mechanisms? Trends Cell Biol 17: 118-126, 2007.

15. Felli N, Fontana L, Pelosi E, et al: MicroRNAs 221 and 222 inhibit normal erythropoiesis and erythroleukemic cell growth via kit receptor down-modulation. Proc Natl Acad Sci USA 102: 18081-18086, 2005.

16. Li J, Huang H, Sun L, et al: MiR-21 indicates poor prognosis in tongue squamous cell carcinomas as an apoptosis inhibitor. Clin Cancer Res 15: 3998-4008, 2009.

17. Wang S, Aurora AB, Johnson BA, Qi X, McAnally J, Hill JA, Richardson JA, Bassel-Duby R and Olson EN: The endothelial-specific microRNA miR-126 governs vascular integrity and angiogenesis. Dev Cell 15: 261-271, 2008.

18. Xu N, Papagiannakopoulos T, Pan G, Thomson JA and Kosik KS: MicroRNA-145 regulates OCT4, SOX2, and KLF4 and represses pluripotency in human embryonic stem cells. Cell 137: 647-658, 2009.

19. Ma L, Young J,Prabhala H, et al: miR-9, a MYC/MYCN-activated microRNA, regulates E-cadherin and cancer metastasis. Nat Cell Biol 12: 247-256, 2010.

20. Huh JH, Kim TH, Kim K, Song JA, Jung YJ, Jeong JY, Lee MJ, Kim YK, Lee DH and An HJ: Dysregulation of miR-106a and miR-591 confers paclitaxel resistance to ovarian cancer. Br J Cancer 109: 452-461, 2013.

21. Fang Y, Shen H, Li H, Cao Y, Qin R, Long L, Zhu X, Xie C and $\mathrm{Xu}$ W: miR-106a confers cisplatin resistance by regulating PTEN/Akt pathway in gastric cancer cells. Acta Biochim Biophys Sin (Shanghai) 45: 963-972, 2013.

22. Xia L, Zhang D, Du R, et al: miR-15b and miR-16 modulate multidrug resistance by targeting BCL2 in human gastric cancer cells. Int J Cancer 123: 372-379, 2008.

23. Fan D, Zhang X, Chen X, Mou Z, Hu J, Zhou S, Ding J and Wu K: Bird's-eye view on gastric cancer research of the past 25 years. J Gastroenterol Hepatol 20: 360-365, 2005.

24. Zhu H, Wu H, Liu X, Evans BR, Medina DJ, Liu CG and Yang JM: Role of MicroRNA miR-27a and miR-451 in the regulation of MDR1/P-glycoprotein expression in human cancer cells. Biochem Pharmacol 76: 582-588, 2008
25. Meng F, Henson R, Lang M, Wehbe H, Maheshwari S, Mendell JT, Jiang J, Schmittgen TD and Patel T: Involvement of human micro-RNA in growth and response to chemotherapy in human cholangiocarcinoma cell lines. Gastroenterology 130 2113-2129, 2006.

26. Zhang CX, Huang S, Xu N, Fang JW, Shen P, Bao YH, Mou BH, Shi MG, Zhong XL and Xiong PJ: Phase II study of epirubicin plus oxaliplatin and infusional 5-fluorouracil as first-line combination therapy in patients with metastatic or advanced gastric cancer. Anticancer Drugs 18: 581-586, 2007.

27. Kopnin BP: Targets of oncogenes and tumor suppressors: Key for understanding basic mechanisms of carcinogenesis. Biochemistry. Biokhimiia 65: 2-27, 2000.

28. Whitmarsh AJ and Davis RJ: Transcription factor AP-1 regulation by mitogen-activated protein kinase signal transduction pathways. J Mol Med Berl 74: 589-607, 1996.

29. Kim HG, Lee CK, Cho SM, Whang K, Cha BH, Shin JH, Song KH and Jeong SW: Neuregulin 1 up-regulates the expression of nicotinic acetylcholine receptors through the ErbB2/ErbB3-PI3K-MAPK signaling cascade in adult autonomic ganglion neurons. J Neurochem 124: 502-513, 2013.

30. Lewis TS, Shapiro PS and Ahn NG: Signal transduction through MAP kinase cascades. Adv Cancer Res 74: 49-139, 1998.

31. Katayama K, Yoshioka S, Tsukahara S, Mitsuhashi J and Sugimoto Y: Inhibition of the mitogen-activated protein kinase pathway results in the down-regulation of P-glycoprotein. Mol Cancer Ther 6: 2092-2102, 2007.

32. Kisucká J, Barancík M, Bohácová V and Breier A: Reversal effect of specific inhibitors of extracellular-signal regulated protein kinase pathway on P-glycoprotein mediated vincristine resistance of L1210 cells. Gen Physiol Biophys 20: 439-444, 2001.

33. Lin JC, Chang SY, Hsieh DS, Lee CF and Yu DS: Modulation of mitogen-activated protein kinase cascades by differentiation-1 protein: Acquired drug resistance of hormone independent prostate cancer cells. J Urol 174: 2022-2026, 2005.

34. Li Y, Li S, Han Y, Liu J, Zhang J, Li F, Wang Y, Liu X and Yao L: Calebin-A induces apoptosis and modulates MAPK family activity in drug resistant human gastric cancer cells. Eur J Pharmacol 591: 252-258, 2008.

35. McCubrey JA, Steelman LS, Abrams SL, Lee JT, Chang F, Bertrand FE, Navolanic PM, Terrian DM, Franklin RA, D'Assoro $\mathrm{AB}$, et al: Roles of the RAF/MEK/ERK and PI3K/PTEN/AKT pathways in malignant transformation and drug resistance. Adv Enzyme Regul 46: 249-279, 2006.

36. Hu Y, Bally M, Dragowska WH and Mayer L: Inhibition of mitogen-activated protein kinase/extracellular signal-regulated kinase kinase enhances chemotherapeutic effects on $\mathrm{H} 460$ human non-small cell lung cancer cells through activation of apoptosis. Mol Cancer Ther 2: 641-649, 2003. 